\title{
In-Situ Polymerization of Isotactic Polypropylene-Nanographite Nanocomposites
}

Brian M. Cromer ${ }^{a}$, Saskia Scheel ${ }^{b}$, Gerrit A. Luinstra ${ }^{b}$, E. Bryan Coughlin $^{a}{ }^{*}$, Alan J. Lesser $^{a, *}$

${ }^{a}$ Department of Polymer Science and Engineering, University of Massachusetts Amherst, 120

Governors Drive Amherst, MA 01003

${ }^{b}$ Institute for Technical and Macromolecular Chemistry, University of Hamburg, Bundesstraße 45, 20146 Hamburg, Germany

* Corresponding Author: Department of Polymer Science and Engineering, University of Massachusetts Amherst, 120 Governors Drive, Amherst, MA 01003-9263. Tel: +1 413577

1316.Email:ajl@polysci.umass.edu (A.J.Lesser),coughlin@mail.pse.umass.edu (E.B. Coughlin).

\begin{abstract}
Isotactic Polypropylene-Exfoliated Graphene Nanoplatelet (iPP-xGnP ${ }^{\mathrm{TM}}$ ) nanocomposites were prepared through an in-situ polymerization technique and compared to analogous composites prepared by melt compounding. In-situ preparation of iPP-xGnP nanocomposites was accomplished via single site metallocene polymerization of propylene within a toluene dispersion of $\mathrm{xGnP}$ nanoparticles. The in-situ prepared nanocomposites were compared to analogous nanocomposites prepared by melt compounding of commercial Ziegler-Natta iPP with xGnP. Optical microscopy showed the in-situ prepared nanocomposites demonstrated poorer $\mathrm{xGnP}$ dispersion compared to composites prepared by melt compounding. All xGnP-reinforced nanocomposites demonstrated increased crystallization temperature, as well as increases in
\end{abstract}


mechanical strength and modulus, relative to neat iPP. However, the non-linear mechanical properties were found to be influenced by the both the preparation method and nanoparticle loading. Nanocomposites prepared by in-situ polymerization generally demonstrated superior ductility and fracture toughness compared to composites prepared by melt compounding. The results are discussed with regard to the preparation technique and xGnP loading.

Key Words: Nanocomposites, Graphite nanoplatelets, Metallocenes, In-situ polymerization, Polyolefins

\subsection{Introduction}

Polyolefin nanocomposites offer opportunities to improve the properties of polyolefins with relatively small amounts of reinforcement. Compared to traditional fiber-reinforced composites, nanocomposites only require small reinforcement concentrations $(<2 \mathrm{vol} \%)$ to create property improvements. Polyolefin nanocomposites have shown property improvements such as mechanical reinforcement, controlled gas permeability, and increased electrical conductivity when compared to the neat polyolefin resins [1].

Many researchers strive to improve the mechanical properties of polyolefins using nanoscale reinforcement in order to create new and economical materials. For example, interest in the automotive industry has been directed toward developing reinforced polyolefins to replace engineering thermoplastic and metallic automotive materials, enabling cost and weight savings [2]. Polyolefin nanocomposites are ideal materials for this application due to the availability of low cost nanoscale reinforcements and polyolefin resins.

Recently, graphene nanoplatelets (GNPs) have been investigated as nanoreinforcements for polyolefins $[3,4]$. The production of GNPs can be achieved by the thermal exfoliation of mineral 
graphite. Most notably, Drzal et al. developed an efficient method to produce Exfoliated Graphene Nanoplatelets $\left(\mathrm{xGnP}^{\mathrm{TM}}\right)$ using acid intercalation followed by microwave assisted exfoliation $[5,6]$. These nanoplatelets are ideal nanoscale reinforcements due to their high aspect ratio, surface area, stiffness, thermal conductivity, and nucleation efficiency for crystallization of polyolefins $[3,4,7,8]$. Typically, polyolefin-GNP nanocomposites demonstrate improved modulus, strength, and higher crystallization temperature, along with decreased strain to failure and fracture toughness, compared to neat polyolefin resins [9]. The apparent decrease in nonlinear mechanical properties has been attributed to the presence of large GNP agglomerates, which can facilitate crack propagation and premature failure.

Accordingly, recent attention has been directed toward developing preparation techniques to improve dispersion in polyolefin-GNP nanocomposites. Melt compounding is the most studied technique to fabricate polyolefin-GNP nanocomposites, however this technique usually does not achieve satisfactory nanoparticle dispersions due to severe aggregation during melt compounding $[4,10,11]$. Solution processing has shown success in other GNP-nanocomposite systems [12,13], however this technique is not applicable to polyolefin-GNP nanocomposites due to the general insolubility of polyolefins. Other fabrication techniques include, nanoparticle surface coating [11], polymerization filling technique [14], Solid State Shear Pulverization [15-17], Solid State Ball Milling [18], and in-situ polymerization [19-23].

In-situ polymerization presents a unique opportunity to prepare well-dispersed polyolefin nanocomposites. This is done by first dispersing nanoparticles in a polymerization solvent, followed by homogeneous polymerization within the dispersion through addition of catalyst and monomer. For polyolefins, homogeneous polymerization is achievable with single site metallocene catalysts [24]. Through rational metallocene catalyst design, features of the 
polyolefin microstructure can be controlled, such as molecular weight, co-monomer incorporation, regio-selectivity, and stereo-selectivity $[25,26]$. In principle, any single site catalyst or combination of catalysts can be applied to an in-situ polymerization, enabling control of the morphology and properties of synthesized PNCs [27,28].

Polyolefin-GNP nanocomposites have recently been prepared via in-situ polymerization, and demonstrated modest increases in linear mechanical properties [19-21,23,29]. However, the non-linear mechanical properties of the PNCs in these studies were limited by their relatively low molecular weight $\left(\mathrm{M}_{\mathrm{w}}<80,000 \mathrm{~g} / \mathrm{mol}\right)$. Accordingly, direct comparisons of the non-linear mechanical properties of in-situ prepared nanocomposites and conventionally melt compounded nanocomposites have not been demonstrated.

The aim of the current study is to prepare comparable iPP-xGnP nanocomposites by two different techniques, and then investigate how the preparation method affects the properties. Therefore, iPP-xGnP nanocomposites were prepared by metallocene catalyzed in-situ polymerization within a toluene dispersion of $\mathrm{xGnP}$, and compared to analogous composites prepared by melt compounding of commercial Ziegler Natta iPP with $\mathrm{xGnP}$ in a static mixer. Also presented is a comprehensive dispersion study at micrometer and nanometer length scales. The relationships between composite morphology and thermal/mechanical properties are discussed.

\subsection{Materials and Methods}

Exfoliated Graphene Nanoplatelets (xGnP-c-750, $750 \mathrm{~m}^{2} / \mathrm{g}$ ) were purchased from XGSciences, Inc and used as received. The preparation method for $\mathrm{xGnP}$ involves microwave assisted exfoliation of intercalated graphite, detailed elsewhere [6]. Polypropylene (PP9999SS) was provided by ExxonMobil. Irganox 1010 and Irgafos 168 were purchased from Ciba and 
used as received. Propylene gas (99.95\%) was provided by Westfalen AG and purified by passage through columns of BASF R3-11G oxygen scavenger and $4 \AA$ molecular sieves. Toluene was provided by Riedel-de-Haën and purified by passage through columns of BASF R3-11G oxygen scavenger and $4 \AA$ molecular sieves. Methylaluminoxane (MAO) was provided by Compton $\mathrm{GmbH}$ and used as received. The metallocene catalyst rac-dimethylsilylbis(2methyl-4-phenyl-1-indenyl)zirconium dichloride ( $\mathrm{rac}-\mathrm{Me}_{2} \mathrm{Si}(2-\mathrm{Me}-4-\mathrm{Ph}-1-\mathrm{Ind})_{2} \mathrm{ZrCl}_{2}$ ) was purchased from Precious Catalyst Inc. and used as received.

\subsection{In-situ Synthesis of iPP-xGnP Composites}

Syntheses were performed in a $9.5 \mathrm{~L}$ steel reactor equipped with a mass flow controller, temperature control system, and mixing blade. Prior to polymerization, $\mathrm{xGnP}$ was added to the reactor and heated to $90{ }^{\circ} \mathrm{C}$ for 12 hours. The reactor was flushed with argon 3 times, and then cooled to $30{ }^{\circ} \mathrm{C}$. Toluene was introduced under constant stirring, followed by MAO. The solution was allowed to equilibrate at $30{ }^{\circ} \mathrm{C}$ for 10 minutes, and then saturated with 3 bars propylene gas under constant mixing (120 RPM). To initiate the reaction, a desired volume of metallocene catalyst solution was injected into the reactor. The temperature and pressure were maintained at $30{ }^{\circ} \mathrm{C}$ and 3 bars propylene gas for the duration of the reaction.

Isotactic polypropylene was also synthesized in a $100 \mathrm{~mL}$ glass reactor with improved temperature control, detailed elsewhere [30]. Toluene, $\mathrm{MAO}$, and $r a c-\mathrm{Me}_{2} \mathrm{Si}(2-\mathrm{Me}-4-\mathrm{Ph}-1-$ Ind) ${ }_{2} \mathrm{ZrCl}_{2}$, were added to the glass reactor within a dry nitrogen glovebox and mixed for 10 minutes. The reactor was then sealed and connected to a gas manifold via Swagelock ${ }^{\circledR}$ QC Series quick connect lines. The solution was under constant magnetic stirring, and the glass 
reactor was placed in a $5 \mathrm{~L}, 30{ }^{\circ} \mathrm{C}$ water bath. The reactor was charged with propylene gas by 15 purge cycles from 0 to 3 bars, and then pressurized to 3 bars propylene gas.

All reactions were terminated by the addition of $10 \mathrm{~mL}$ of $5 \mathrm{wt} \% \mathrm{HCl}$ in ethanol. The products were washed in $2 \mathrm{~L}$ dilute hydrochloric acid, stirred overnight, and rinsed thoroughly with ethanol. Finally, the products were dried under vacuum at $60{ }^{\circ} \mathrm{C}$ for 48 hours. The products were fine reactor powders, seen in Figure 1.

\subsection{Melt Compounding of iPP-xGnP composites}

Melt compounding of iPP-xGnP nanocomposites was performed in a $50 \mathrm{~mL}$ Brabender static mixer (R. E. E. 6) operating at $200{ }^{\circ} \mathrm{C}$ for 15 minutes at 70 RPM. Commercial Ziegler-Natta isotactic polypropylene (ExxonMobil, PP9999SS), $\mathrm{xGnP}$, and $0.1 \mathrm{wt} \%$ process stabilizers (Irganox 1010 and Irgafos 168) were dry-mixed in a plastic bag prior to addition to the mixer.

\subsection{Characterization Methods}

The infrared absorption profile of $\mathrm{xGnP}$ was characterized with a Perken Elmer Spectra 100 FTIR. A $0.04 \mathrm{wt} \% \mathrm{xGnP}$ in $\mathrm{KBr}$ pellet was prepared and then analyzed in transmission mode. The surface chemistry of xGnP was analyzed using XPS, using a PHI Quantum 2000 Scanning ESCA Microprobe with monochromatic Al Ka radiation. A $200 \mu \mathrm{m}^{2}$ spot was selected for analysis. A low take off angle of $10^{\circ}$ was used to avoid the mounting material. Samples were analyzed with low resolution survey spectra of pass energy $187.9 \mathrm{eV}$ as well as multiplexes at $46.95 \mathrm{eV}$ for quantitative analysis. Prior to analysis, samples were dried at $100{ }^{\circ} \mathrm{C}$ in a vacuum oven for 12 hours. Optical characterization was conducted on an Olympus optical microscope with DP71 digital camera. Film samples (50 $\mu \mathrm{m}$ thick) were prepared by compression molding at $200{ }^{\circ} \mathrm{C}$. Samples were analyzed in transmission mode. Transmission electron micrographs of 
composites were obtained with a JEOL JEM-2000FX transmission electron microscope with $\mathrm{LaB}_{6}$ electron source, at accelerating voltage $200 \mathrm{kV}$. Thin $(\sim 40 \mathrm{~nm})$ sample sections were prepared using a Leica CryoUltramicrotome and Microstar diamond knife and then imaged on 400 mesh copper grids. Polypropylene molecular weight was characterized with hightemperature gel permeation chromatography on a Polymer Labs PL-220 GPC. Polypropylene was removed from the composites via Soxhlet extraction in 1,2,4 Trichlorobenzene for 12 hours. Samples were then dissolved and analyzed in $1,2,4$ Trichlorobenzene at $145^{\circ} \mathrm{C}$ against polystyrene standards. Molecular weights were converted from polystyrene to polypropylene using the universal calibration method based on the Mark-Houwink constants. Thermal properties were measured using a Thermal Gravimetric Analyzer (TA Instruments-TGA Q500), and Differential Scanning Calorimeter (TA Instruments-DSC Q200). TGA was conducted in a nitrogen atmosphere, and heated to $600{ }^{\circ} \mathrm{C}$ at $10{ }^{\circ} \mathrm{C} / \mathrm{min}$. DSC was performed between 20 and $200{ }^{\circ} \mathrm{C}$ at $10{ }^{\circ} \mathrm{C} / \mathrm{min}$. Crystallization and melting analyses were performed on the first cooling and the second heating cycle, respectively. Melting and cooling enthalpies for each sample were normalized to iPP mass [31]. To prepare samples for mechanical analysis, reactor powders were dry mixed with $0.1 \mathrm{wt} \%$ process stabilizers (Irganox 1010 and Irgafos 168) prior to compression molding. Square plaques were compression molded at $200{ }^{\circ} \mathrm{C}$ for $8 \mathrm{~min}$ and then cooled at 30 $\mathrm{K} / \mathrm{min}$. Tensile specimens were milled from $3 \mathrm{~mm}$ thick plaques. Tensile properties were characterized according to ASTM D 638. Testing was performed at room temperature using an Instron 4466 testing machine at a crosshead speed of $2 \mathrm{~mm} / \mathrm{min}$ and a preload force of $8 \mathrm{~N}$. The strain was calculated from the crosshead displacement. Plane strain fracture toughness was characterized according to ASTM D5045 in a single-edge-notch three point bend configuration. Samples were milled from $6 \mathrm{~mm}$ thick plaques and pre-notched with a diamond saw. A natural 
crack was made by cooling the sample to $-20{ }^{\circ} \mathrm{C}$ and displacing a fresh razor $1.5 \mathrm{~mm}$ into each sample with an Instron 4455 testing machine.

\subsection{Results and Discussion}

Surface characterization of $\mathrm{xGnP}$ was performed to identify functional groups that could potentially inhibit the in-situ polymerization reaction. Both FTIR and XPS suggest oxidized carbon functional groups are present. The FTIR absorptions from $1580-1710 \mathrm{~cm}^{-1}$ and the asymmetric absorption at $3438 \mathrm{~cm}^{-1}$ indicate carboxylic acids are present. The weak absorptions from 1100-1250 $\mathrm{cm}^{-1}$ suggest a range of tertiary and/or aromatic alcohols (SI-1). From XPS, the carbon peak demonstrates a maximum near $284 \mathrm{eV}$, corresponding to graphitic C-C bonds. Also shown is a weak feature near $288 \mathrm{eV}$, corresponding to carbonyl-bearing functional groups such as carboxylic acids, ketones, and aldehydes. The C:O ratio was determined to be 10.6:1 (SI-2). While the precise chemical structure of the functional groups are difficult to ascertain due to the heterogeneous nature of $\mathrm{xGnP}$, it is clear that active hydrogen-bearing functional groups $(-\mathrm{OH}$, $\mathrm{C}=\mathrm{O}$, and $-\mathrm{COOH})$ are present. These oxidized functional groups are caused by the preparation process for $\mathrm{xGnP}$, which induces oxidation through strongly acidic intercalation compounds and extreme exfoliation temperatures [12]. Additionally, mineral graphite is known to contain $\mathrm{sp}^{3}$ hybridized carbon defects that are amenable to oxidation during the exfoliation process [32].

\subsection{In-Situ Synthesis of iPP-xGnP Nanocomposites}

Isotactic Polypropylene-xGnP (iPP-xGnP) nanocomposites were prepared using metallocene catalyzed polymerization of polypropylene within a toluene suspension of xGnP nanoparticles. The high activity catalyst, $r a c-\mathrm{Me}_{2} \mathrm{Si}(2-\mathrm{Me}-4-\mathrm{Ph}-1-\mathrm{Ind})_{2} \mathrm{ZrCl}_{2}$, is a $C_{2}$-symmetric zirconocene designed to polymerize high molecular weight isotactic polypropylene $[33,34]$. Like other $C_{2^{-}}$ 
symmetric zirconocenes, the performance of $r a c-\mathrm{Me}_{2} \mathrm{Si}(2-\mathrm{Me}-4-\mathrm{Ph}-1-\mathrm{Ind})_{2} \mathrm{ZrCl}_{2}$ is a function of temperature. The molecular weight of the polypropylene synthesized from this catalyst decreases with increasing reaction temperature, while the catalyst activity increases with increasing reaction temperature. For this study, the optimal reaction temperature for high molecular weight iPP with modest activity was $30{ }^{\circ} \mathrm{C}$. Other studies used higher reaction temperatures, which yielded higher polymerization activity with lower molecular weight [20,21]. High Methylaluminoxane (MAO) concentration and high Al:Zr ratio were selected in order to passivate the active hydrogens on $\mathrm{xGnP}$ prior to contact with the metallocene catalyst. Accordingly, $\mathrm{xGnP}$ was pretreated with excess $\mathrm{MAO}$ for 10 minutes prior to contact with rac$\mathrm{Me}_{2} \mathrm{Si}$ (2-Me-4-Ph-1-Ind) ${ }_{2} \mathrm{ZrCl}_{2}$. When $\mathrm{xGnP}$ was pretreated with this method, catalyst activity was acceptable $\left(\geq 2 \mathrm{~kg} \mathrm{mmol}^{-1} \mathrm{bar}^{-1} \mathrm{hr}^{-1}\right)$ for all samples. Figure 1 shows the color of each sample, which becomes progressively darker with increasing xGnP concentration. Table 1 summarizes the reaction parameters of the $i n-s i t u$ synthesized iPP-xGnP nanocomposites. In-situ synthesized samples are named "IS- $n$," where $n$ is the weight percent of xGnP determined by the char yield after TGA. Similarly, melt compounded samples are named "MC- $n$." 


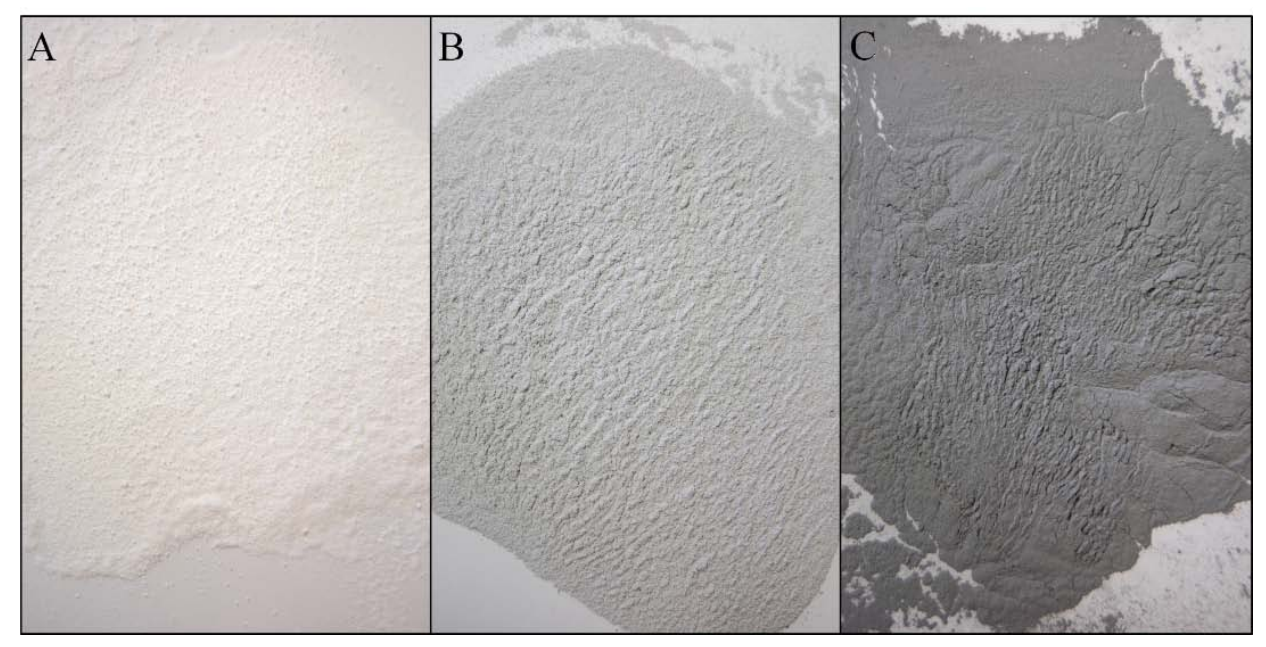

Figure 1: Reactor powder from in-situ synthesis of iPP-xGnP nanocomposites. (A) IS-0 (B) IS-2 (C) IS-4. (1.5 Column Figure)

Table 1 shows an apparent decrease in the catalyst activity with increasing $\mathrm{xGnP}$ concentration. This trend is ascribed to both reaction temperature fluctuations and catalyst poisoning from the surface chemistry on $\mathrm{xGnP}$. Temperature fluctuations are anticipated during propylene polymerization, a highly exothermic reaction generating $\sim 89 \mathrm{~kJ} / \mathrm{mol}$ propylene. For this reason, the large $9.5 \mathrm{~L}$ reaction vessel used to polymerize samples IS-0 through IS-4 was fitted with a jacketed cooling system, and the smaller $20 \mathrm{~mL}$ reactor used to polymerize sample IS-0_20mL was placed in a $30{ }^{\circ} \mathrm{C}$ water bath. Nonetheless, heat transfer is a significant operational challenge in the $9.5 \mathrm{~L}$ reactor, so the actual polymerization temperature is likely higher than the setpoint temperature $\left(30^{\circ} \mathrm{C}\right)$. Consequently, samples IS-0 through IS-4 demonstrate higher catalyst activity than sample IS-0_20 mL. Also, the catalyst activity decreases with increasing $\mathrm{xGnP}$ concentration. In fact, sample IS-4 required polymerization at $40{ }^{\circ} \mathrm{C}$ in order to achieve sufficient catalyst activity. The observed decrease in catalyst activity suggests that $\mathrm{xGnP}$ has a deleterious effect on the metallocene catalyst and/or MAO cocatalyst, possibly due to the active hydrogen bearing functional groups on $\mathrm{xGnP}$. 
Table 1

Reaction parameters and properties of iPP-xGnP nanocomposites. $T p=$ polymerization temperature set point. Propylene pressure $=3$ bars. *kg iPP mmol Zr-1 bar-1 h-1. **Mw/Mn (Double Column Figure)

\begin{tabular}{|c|c|c|c|c|c|c|c|c|c|c|c|c|c|c|c|}
\hline Sample ID & $\begin{array}{c}{[\mathrm{MAO}]} \\
(\mathrm{mM})\end{array}$ & $\begin{array}{c}T_{p} \\
\left({ }^{\circ} \mathrm{C}\right)\end{array}$ & $\begin{array}{l}\mathrm{Al}: \mathrm{Zr} \\
\text { ratio }\end{array}$ & $\begin{array}{l}\text { Volume } \\
\text { (L) }\end{array}$ & Activity* & $\begin{array}{c}M_{w} \\
(\mathrm{kDa})\end{array}$ & $\mathrm{D}^{* *}$ & $\begin{array}{c}T_{C} \\
\left({ }^{\circ} \mathrm{C}\right)\end{array}$ & $\begin{array}{c}T_{M} \\
\left({ }^{\circ} \mathrm{C}\right)\end{array}$ & $\begin{array}{c}X_{C} \\
(\%)\end{array}$ & $\begin{array}{c}\text { Char } \\
\text { Yield (\%) }\end{array}$ & $E(\mathrm{MPa})$ & $\begin{array}{c}\sigma_{y} \\
\text { (MPa) }\end{array}$ & $\varepsilon_{b}(\%)$ & $G_{I C}\left(\mathrm{~kJ} / \mathrm{m}^{2}\right)$ \\
\hline IS-0 & 0.03 & 30 & $7,000: 1$ & 5 & 9.48 & 141 & 2.35 & 114.6 & 157.0 & 50.7 & 0.0 & 1620.0 & 38.4 & 12.1 & 14.6 \\
\hline IS-2 & 0.1 & 30 & $50,000: 1$ & 5 & 5.43 & 252 & 2.71 & 125.3 & 160.0 & 49.5 & 1.9 & 1830.0 & 38.5 & 78.0 & 16.2 \\
\hline IS-4 & 0.1 & 40 & $50,000: 1$ & 5 & 2.09 & 137 & 2.39 & 127.0 & 160.0 & 51.4 & 3.9 & 2000.0 & 43.0 & 5.8 & 6.3 \\
\hline IS- $0 \_20 \mathrm{~mL}$ & 0.03 & 30 & 7,000:1 & 0.02 & 1.93 & 1,189 & 2.07 & - & - & - & - & - & - & - & - \\
\hline MC-0 & - & - & - & - & - & 135 & 3.55 & 111.7 & 164.0 & 50.7 & 0.0 & 1050.0 & 33.2 & 11.7 & 4.0 \\
\hline MC-2 & - & - & - & - & - & 135 & 3.55 & 130.4 & 165.5 & 58.0 & 2.0 & 1100.0 & 34.6 & 7.0 & 0.5 \\
\hline MC-4 & - & - & - & - & - & 135 & 3.55 & 132.8 & 166.0 & 59.6 & 4.0 & 1450.0 & 35.3 & 4.0 & 0.4 \\
\hline
\end{tabular}


The trend in molecular weight and $Đ$ is also ascribed to temperature fluctuations. Sample IS$0 \_20 \mathrm{~mL}$ showed the highest molecular weight and lowest $Đ$, due to efficient heat transfer during this small scale polymerization. In contrast, sample IS-0 had a much lower molecular weight and broader $Ð$ due to heat transfer challenges. When $\mathrm{xGnP}$ is present, the activity is attenuated to a manageable level, thus minimizing temperature fluctuations. The result is that the molecular weight of IS-2 is higher than IS-0. Sample IS-4 had the lowest molecular weight due to the high polymerization temperature necessary to synthesize this sample.

\subsection{Morphology of iPP-xGnP Nanocomposites}

The micron-scale dispersion of $\mathrm{xGnP}$ particles in iPP was directly imaged with transmission optical microscopy (Figure 2). The melt compounded sample series (MC-) demonstrate smaller agglomerate size compared to the in-situ synthesized sample series (IS-). Both composites possess numerous agglomerates of apparent diameter 1-10 $\mu \mathrm{m}$; however the IS- sample series contained several large $(>10 \mu \mathrm{m})$ agglomerates. The large $\mathrm{xGnP}$ agglomerates seen in the ISsample series are attributed to the unfavorable nanoparticle-polymerization solvent interaction, as well as insufficient mixing conditions during polymerization. It has been shown that the poor interaction energy between graphitic nanoparticles and toluene promotes rapid nanoparticle flocculation and sedimentation [35]. Accordingly, in-situ polymerization is performed with mechanical agitation in order to promote dispersive mixing of $x \mathrm{GnP}$ nanoparticles. However, the

presence of large agglomerates in the IS- sample series suggests that the mixing flow field during in-situ polymerization cannot overcome the sedimentation kinetics, likely because it is difficult to achieve sufficient mixing shear stresses in a low viscosity polymerization solvent. In contrast, 
melt compounding in molten iPP promoted more fragmentation due to the comparatively higher mixing viscosity, and therefore shear stress.

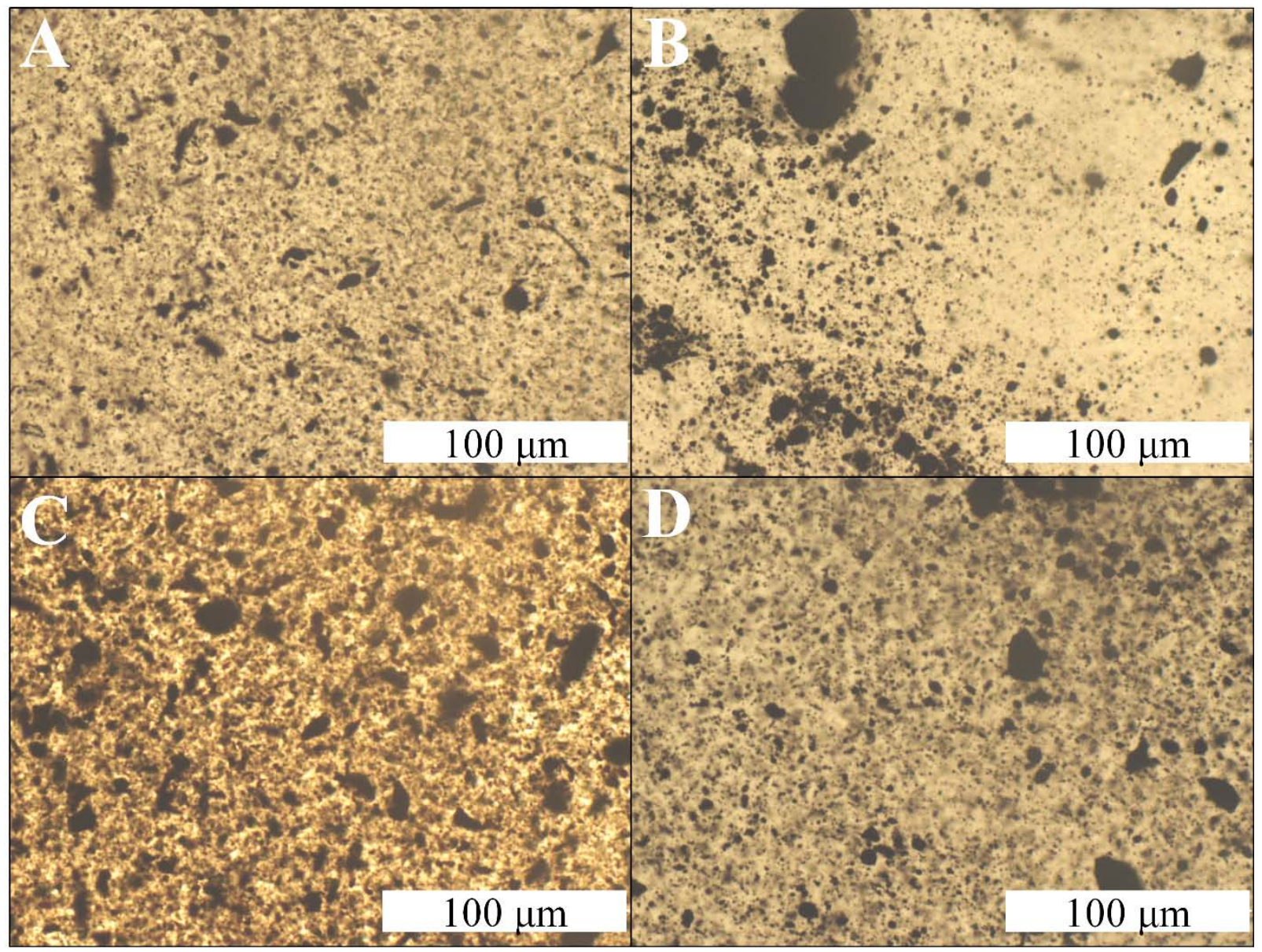

Figure 2: Transmission optical microscopy images of $50 \mu \mathrm{m}$ thick iPP-xGnP films. (A) MC-2 (B) IS-2 (C) MC-4 (D) IS-4. (1.5 Column Figure)

The nano-scale dispersion of $\mathrm{xGnP}$ in iPP was directly evaluated with TEM (Figure 3). IS-0 shows several $\sim 20 \mathrm{~nm}$ diameter spherical particles, which are ascribed to residual $\mathrm{Al}_{2} \mathrm{O}_{3}$ particles from MAO hydrolysis. IS-2 and IS-4 show large agglomerates of 1-5 microns in diameter, each comprised of several $\mathrm{xGnP}$ platelets. The results suggest that the nanoscale dispersion of $\mathrm{xGnP}$ is poor for all samples. Interestingly, IS-2 and IS-4 did not contain $\mathrm{Al}_{2} \mathrm{O}_{3}$ artifacts from $\mathrm{MAO}$ 
hydrolysis, even though the MAO concentration was 3x larger than IS-0. One possible explanation could be that the MAO hydrolysis byproducts are confined to the $\mathrm{xGnP}$ surface.

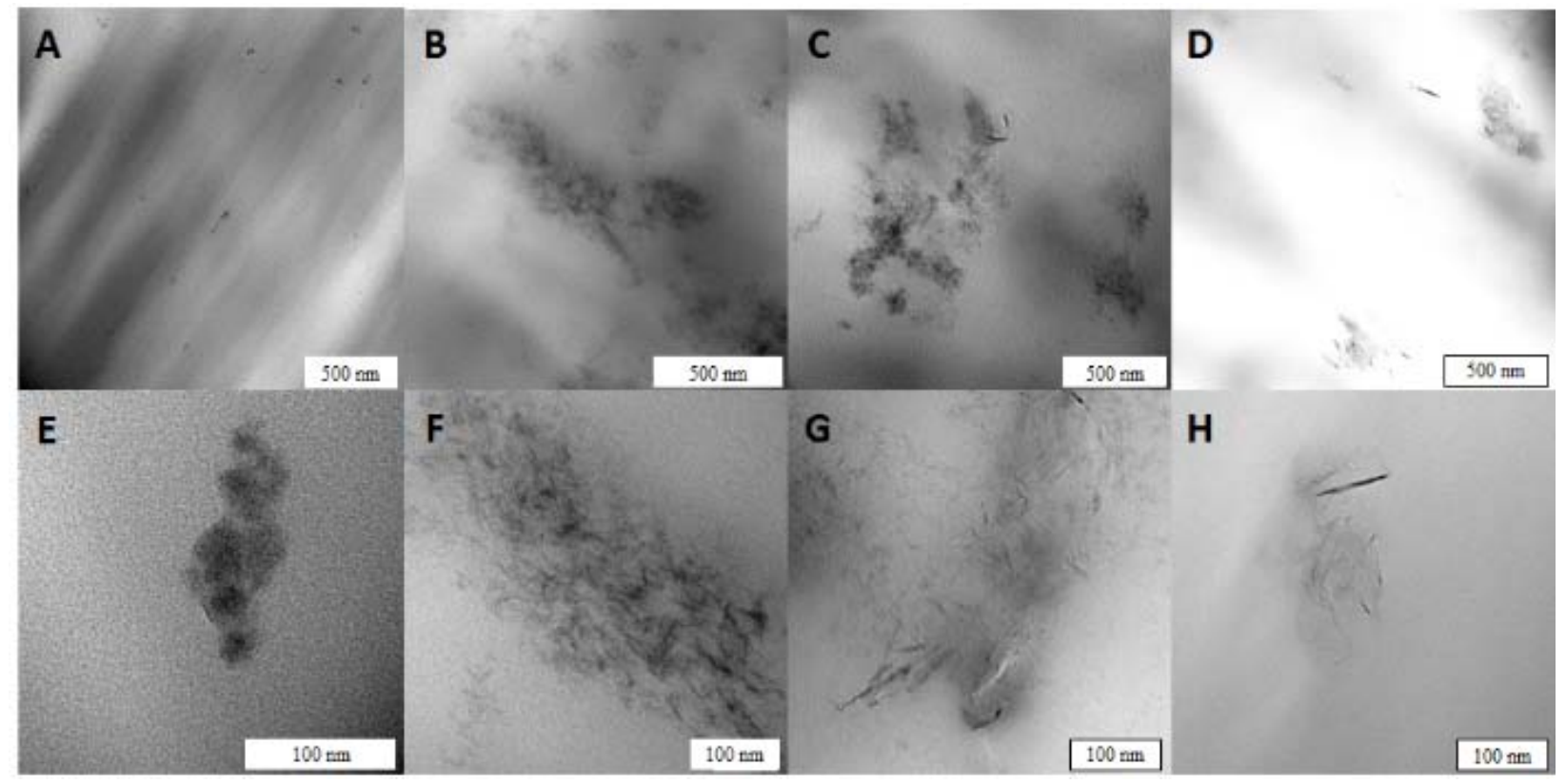

Figure 3: Transmission electron microscopy images of iPP-xGnP films. (A,E) IS-0 (B,F) IS-2 (C,G) IS-4 (D,H) MC-2. (Double Column Figure)

Thermal calorimetry was used as an indirect method to characterize the $\mathrm{xGnP}$ dispersion in iPP. Exfoliated graphene nanoplatelets are known nucleating agents for iPP [8]. For iPP-xGnP nanocomposites, the crystallization temperature $\left(T_{c}\right)$ is expected to increase with increasing $\mathrm{xGnP}$ loading and/or improved dispersion. Indeed, thermal calorimetry shows increased $T_{c}$ with increasing $\mathrm{xGnP}$ loading for all samples (Table 1, Figure 4A). The increase relative to neat resin is greater for the MC- sample series (+21.1 K) compared to the IS- sample series $(+12.4 \mathrm{~K})$, suggesting melt compounding produces better $\mathrm{xGnP}$ dispersions than in-situ synthesis. 

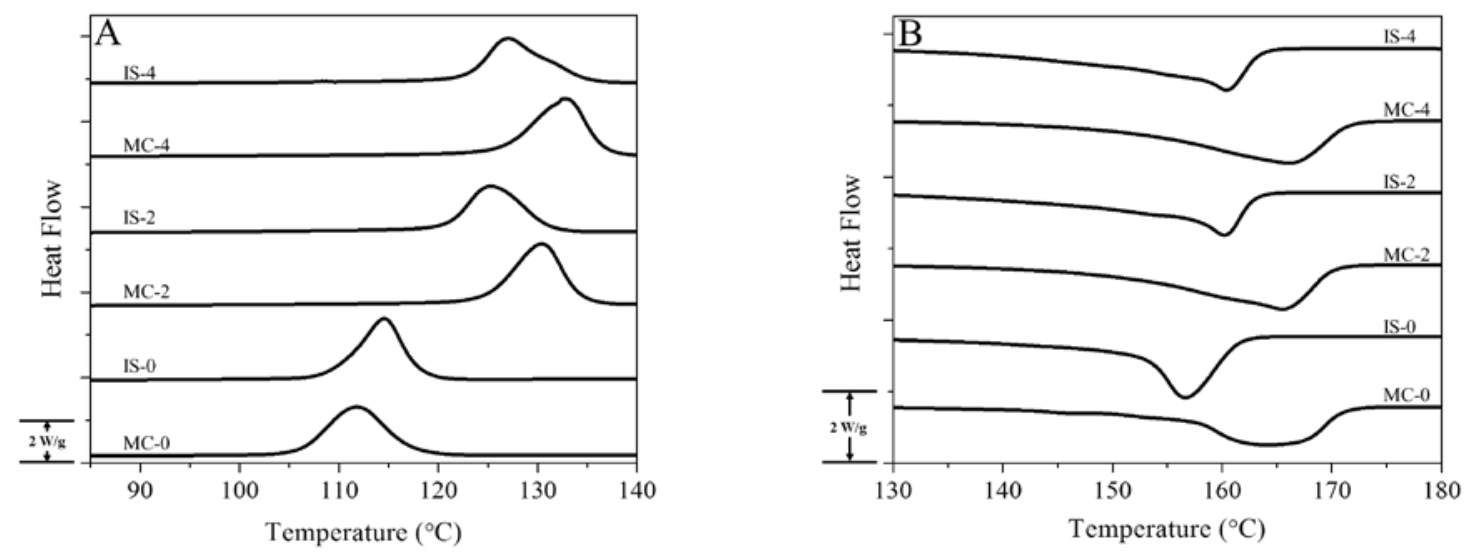

Figure 4: Differential Scanning Calorimetry of IS- and MC-samples. (A) Cooling and (B) heating curves.

Thermal calorimetry also reveals information about other morphological features such as crystal volume fraction and lamellar thickness. Both the IS- and MC-sample series demonstrated increasing melting point $\left(T_{m}\right)$ and crystal volume fraction $(X c)$ with increasing xGnP concentration (Table 1, Figure 4B). However, the melting points of the IS- samples are less than those of the MC-samples, which is attributed to the tendency of metallocene iPP to have a higher concentration of regio- and stereo-defects, as well as a higher regio- to stereodefect ratio in the chain microstructure, compared to Ziegler-Natta iPP [25,36-39]. These defects in chain microstructure ultimately decreases the iPP lamellar crystal thickness, and therefore $T_{m}$.

\subsection{Mechanical Characterization}

Tensile tests were performed to ascertain the effect of xGnP loading and preparation method on the linear and non-linear mechanical properties of iPP-xGnP nanocomposites. Both the ISand MC-sample series showed modest increases in Young's modulus and yield stress with increasing xGnP loading, a common observation in nanocomposites [40]. While it is difficult to precisely determine the physical origin of nanoreinforcement, the increases in modulus and 
strength in the present study are likely due to a combination of factors. Nucleating agents like $\mathrm{xGnP}$ will impart an increase in stiffness by changing the iPP crystal morphology and augmenting the crystal volume fraction. Moreover, the interfacial interaction between $\mathrm{xGnP}$ and the iPP matrix may affect the segmental mobility of iPP chains, contributing to reinforcement [41]. In addition in reinforcement, both the IS- and MC- sample series showed decreased ductility with increasing $\mathrm{xGnP}$ loading. A decrease in ductility relative to neat resin is also commonly observed in nanoparticle reinforced systems and has been attributed to the structural flaws created by agglomerates of nanoparticles [4,9]. The IS- sample series demonstrates increased ductility compared to the MC-sample series, which is ascribed to the differences in chain microstructure of metallocene iPP and Ziegler-Natta iPP, specifically the concentration and type of regio- and stereo-defects [25]. Interestingly, IS-2 showed the largest elongation at break, likely due to its comparatively high molecular weight. The results suggest that the correct combination of molecular weight and chain microstructure can promote ductility, even in the presence of structural flaws. Table 1 and Figure 5 summarize the effect of $x \mathrm{GnP}$ and preparation method on the mechanical properties of iPP-xGnP composites. 


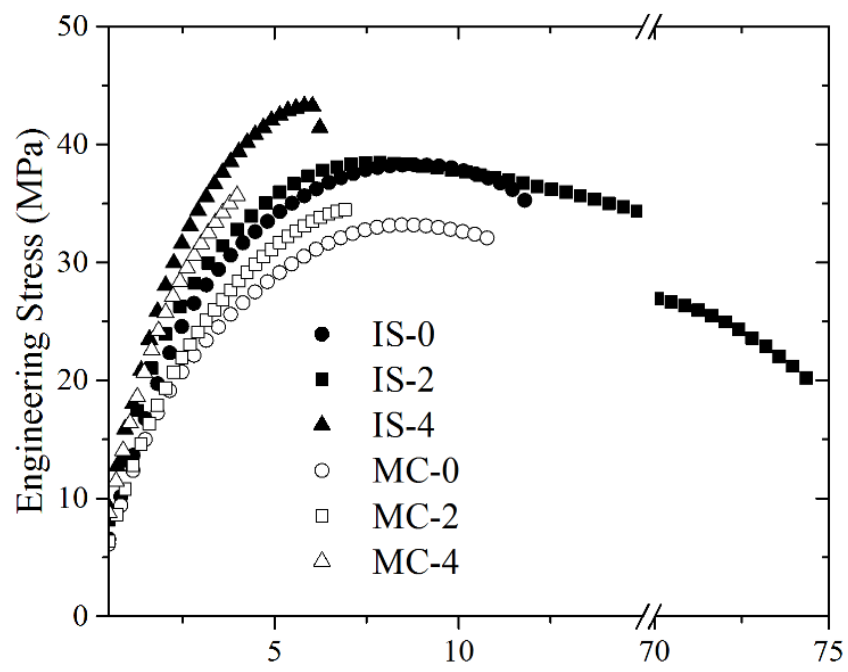

Tensile Strain (\%)

Figure 5: Tensile properties of iPP-xGnP nanocomposites. (Single Column Figure)

The effect of $x \mathrm{GnP}$ nanoreinforcement on the toughness of iPP-xGnP nanocomposites was determined by plane strain fracture toughness tests in the single edge notched 3-point bend (SENB) configuration. Generally, samples with $\mathrm{xGnP}$ reinforcement showed decreased critical strain energy release rate $\left(\mathrm{G}_{1 \mathrm{C}}\right)$ relative to the neat resin. The decrease in toughness is attributed to large $(>10 \mu \mathrm{m}) \mathrm{xGnP}$ agglomerates, which are expected to decrease fracture toughness by creating flaws in the iPP matrix (Figure 2). However, IS-2 showed improved fracture toughness relative to the neat resin. This is likely due to the comparatively high molecular weight of this sample. The melt compounded samples showed the largest decrease in fracture toughness ( $\sim 90 \%$ decrease) relative to MC-0, while the in-situ synthesized composites maintained more toughness ( $\sim 60 \%$ decrease) relative to IS-0. Furthermore, the IS- sample series demonstrated significantly improved fracture toughness compared to the MC-sample series, which is again attributed to the differences in chain microstructure of Metallocene iPP and Ziegler-Natta iPP 
[25]. The results suggest that molecular weight, chain microstructure and $\mathrm{xGnP}$ loading affect the non-linear mechanical response of iPP-xGnP nanocomposites (Table 1).

\subsection{Conclusions}

Nanocomposites of Exfoliated Graphene Nanoplatelets ( $\left.\mathrm{xGnP}{ }^{\circledR}\right)$ and isotactic polypropylene (iPP) are prepared via in-situ metallocene polymerization of propylene within a solution dispersion of $\mathrm{xGnP}$ nanoparticles. The in-situ prepared nanocomposites were compared to analogous composites of similar molecular weight, prepared by melt compounding of commercial Ziegler-Natta iPP and $\mathrm{xGnP}$. The in-situ polymerization requires excess $\mathrm{MAO}$ to prevent $\mathrm{xGnP}$ from poisoning the metallocene catalyst. Dispersion analysis by optical and transmission electron microscopy shows the melt compounding technique produces nanocomposites with finer $\mathrm{xGnP}$ dispersions than the in-situ polymerization technique. All composites demonstrate improved mechanical modulus relative to neat iPP. However, the in-situ prepared composites demonstrate superior non-linear mechanical properties such as ductility and toughness, likely due to the differences in chain microstructure of metallocene-iPP and commercial Ziegler-Natta-iPP. The results are discussed with regard to the preparation method, molecular weight, chain microstructure, and $\mathrm{xGnP}$ loading.

\section{Acknowledgements}

Financial support was provided by the Department of Defense (DoD) through the National Defense Science \& Engineering Graduate Fellowship (NDSEG) awarded to B. M. Cromer. Funding was also provided by the Center for UMass/Industry Research on Polymers (CUMIRP)

Cluster M: Mechanics of Polymers and Composites and Cluster F: Fire Safe Polymers and 
Polymer Composites. We also acknowledge Professor L. Drzal for kindly providing xGnP materials.

\section{References}

[1] Vaia RA, Wagner HD. Framework for nanocomposites. Mater Today 2004;7:32-7.

[2] Robert Eller. Current States and Future Potential for Polyolefins, TPOs, and TPEs in the Global Automotive Market. Houston, TX: 2012.

[3] Jang BZ, Zhamu A. Processing of nanographene platelets (NGPs) and NGP nanocomposites: a review. J Mater Sci 2008;43:5092-101.

[4] Li B, Zhong W-H. Review on polymer/graphite nanoplatelet nanocomposites. J Mater Sci 2011;46:5595-614.

[5] Fukushima. H. Graphite Nanoreinforcements in Polymer Nanocomposites. Michigan State University, 2003.

[6] Lawrence T. Drzal HF. Expanded graphite and products produced therefrom. US8501858 B2, 2013.

[7] Park S, Ruoff RS. Chemical methods for the production of graphenes. Nat Nanotechnol 2009;4:217-24.

[8] Kalaitzidou K, Fukushima H, Askeland P, Drzal LT. The nucleating effect of exfoliated graphite nanoplatelets and their influence on the crystal structure and electrical conductivity of polypropylene nanocomposites. J Mater Sci 2007;43:2895-907.

[9] Kalaitzidou K, Fukushima H, Drzal LT. A new compounding method for exfoliated graphite-polypropylene nanocomposites with enhanced flexural properties and lower percolation threshold. Compos Sci Technol 2007;67:2045-51.

[10] Zheng W, Lu X, Wong S-C. Electrical and mechanical properties of expanded graphitereinforced high-density polyethylene. J Appl Polym Sci 2004;91:2781-8.

[11] Jiang X, Drzal LT. Improving electrical conductivity and mechanical properties of high density polyethylene through incorporation of paraffin wax coated exfoliated graphene nanoplatelets and multi-wall carbon nano-tubes. Compos Part A Appl Sci Manuf 2011;42:1840-9.

[12] Zheng W, Wong S-C. Electrical conductivity and dielectric properties of PMMA/expanded graphite composites. Compos Sci Technol 2003;63:225-35. 
[13] Zheng W, Wong S-C, Sue H-J. Transport behavior of PMMA/expanded graphite nanocomposites. Polymer 2002;43:6767-73.

[14] Stürzel M, Kempe F, Thomann Y, Mark S, Enders M, Mülhaupt R. Novel Graphene UHMWPE Nanocomposites Prepared by Polymerization Filling Using Single-Site Catalysts Supported on Functionalized Graphene Nanosheet Dispersions. Macromolecules 2012;45:6878-87.

[15] Wakabayashi K, Pierre C, Dikin DA, Ruoff RS, Ramanathan T, Brinson LC, et al. Polymer-Graphite Nanocomposites: Effective Dispersion and Major Property Enhancement via Solid-State Shear Pulverization. Macromolecules 2008;41:1905-8.

[16] Torkelson JM, Wakabayashi K. Polymer-graphite nanocomposites via solid-state shear pulverization. US7906053 B1, 2009.

[17] Wakabayashi K, Brunner PJ, Masuda J, Hewlett SA, Torkelson JM. Polypropylenegraphite nanocomposites made by solid-state shear pulverization: Effects of significantly exfoliated, unmodified graphite content on physical, mechanical and electrical properties. Polymer 2010;51:5525-31.

[18] Jiang X, Drzal LT. Reduction in percolation threshold of injection molded high-density polyethylene/exfoliated graphene nanoplatelets composites by solid state ball milling and solid state shear pulverization. J Appl Polym Sci 2012;124:525-35.

[19] Fim F de C, Guterres JM, Basso NRS, Galland GB. Polyethylene/graphite nanocomposites obtained by in situ polymerization. J Polym Sci Part A Polym Chem 2010;48:692-8.

[20] Milani MA, Quijada R, Basso NRS, Graebin AP, Galland GB. Influence of the graphite type on the synthesis of polypropylene/graphene nanocomposites. J Polym Sci Part A Polym Chem 2012;50:3598-605.

[21] Milani MA, González D, Quijada R, Basso NRS, Cerrada ML, Azambuja DS, et al. Polypropylene/graphene nanosheet nanocomposites by in situ polymerization: Synthesis, characterization and fundamental properties. Compos Sci Technol 2013;84:1-7.

[22] Zou J-F, Yu Z-Z, Pan Y-X, Fang X-P, Ou Y-C. Conductive mechanism of polymer/graphite conducting composites with low percolation threshold. J Polym Sci Part B Polym Phys 2002;40:954-63.

[23] Polschikov S V., Nedorezova PM, Klyamkina AN, Kovalchuk AA, Aladyshev AM, Shchegolikhin AN, et al. Composite materials of graphene nanoplatelets and polypropylene, prepared by in situ polymerization. J Appl Polym Sci 2013;127:904-11.

[24] Resconi L, Cavallo L, Fait A, Piemontesi F. Selectivity in Propene Polymerization with Metallocene Catalysts. Chem Rev 2000;100:1253-346. 
[25] De Rosa C, Auriemma F. Single site metallorganic polymerization catalysis as a method to probe the properties of polyolefins. Polym Chem 2011;2:2155.

[26] Severn JR, Chadwick JC, Duchateau R, Friederichs N. "Bound but not gagged"-immobilizing single-site alpha-olefin polymerization catalysts. Chem Rev 2005;105:4073-147.

[27] Cecchin G, Morini G, Pelliconi A. Polypropene product innovation by reactor granule technology. Macromol Symp 2001;173:195-210.

[28] Böhm LL. The ethylene polymerization with Ziegler catalysts: fifty years after the discovery. Angew Chem Int Ed Engl 2003;42:5010-30.

[29] Shevchenko VG, Polschikov S V., Nedorezova PM, Klyamkina AN, Shchegolikhin AN, Aladyshev AM, et al. In situ polymerized poly(propylene)/graphene nanoplatelets nanocomposites: Dielectric and microwave properties. Polymer 2012;53:5330-5.

[30] Constable GS, Gonzalez-Ruiz RA, Kasi RM, Coughlin EB. Gas Manifold for Olefin Polymerization and a Convenient Reactor Design for the Parallel Screening of Catalysts. Macromolecules 2002;35:9613-6.

[31] Wunderlich B. Macromolecular Physics. New York, NY: Academic Press; 1980.

[32] Kim H, Abdala AA, Macosko CW. Graphene/Polymer Nanocomposites. Macromolecules 2010;43:6515-30.

[33] Spaleck W, Kueber F, Winter A, Rohrmann J, Bachmann B, Antberg M, et al. The Influence of Aromatic Substituents on the Polymerization Behavior of Bridged Zirconocene Catalysts. Organometallics 1994;13:954-63.

[34] Stehling U, Diebold J, Kirsten R, Roell W, Brintzinger HH, Juengling S, et al. ansaZirconocene Polymerization Catalysts with Anelated Ring Ligands - Effects on Catalytic Activity and Polymer Chain Length. Organometallics 1994;13:964-70.

[35] Grujicic M, Cao G, Roy WN. Atomistic simulations of the solubilization of single-walled carbon nanotubes in toluene. J Mater Sci 2004;39:2315-25.

[36] Bond EB, Spruiell JE. The effects of atacticity, comonomer content, and configurational defects on the equilibrium melting temperature of monoclinic isotactic polypropylene. $\mathrm{J}$ Appl Polym Sci 2001;81:229-36.

[37] Janimak JJ, Cheng SZD, Zhang A, Hsieh ET. Isotacticity effect on crystallization and melting in polypropylene fractions: 3 . Overall crystallization and melting behaviour. Polymer 1992;33:728-35. 
[38] Cheng SZD, Janimak JJ, Zhang A, Hsieh ET. Isotacticity effect on crystallization and melting in polypropylene fractions: 1 . Crystalline structures and thermodynamic property changes. Polymer 1991;32:648-55.

[39] Gómez-Elvira JM, Tiemblo P, Elvira M, Matisova-Rychla L, Rychly J. Relaxations and thermal stability of low molecular weight predominantly isotactic metallocene and Ziegler-Natta polypropylene. Polym Degrad Stab 2004;85:873-82.

[40] Jancar J, Douglas JF, Starr FW, Kumar SK, Cassagnau P, Lesser AJ, et al. Current issues in research on structure-property relationships in polymer nanocomposites. Polymer 2010;51:3321-43.

[41] Miltner HE, Assche G Van, Pozsgay A, Pukánszky B, Mele B Van. Restricted chain segment mobility in poly(amide) 6/clay nanocomposites evidenced by quasi-isothermal crystallization. Polymer 2006;47:826-35. 
